\title{
Diferencias de género en la valoración de los atributos del pisco
}

\author{
Gender differences in the assessment of pisco attributes
}

Sebastián Araya-Pizarro*1, Enrique Ruiz-Vega ${ }^{1}$

\begin{abstract}
RESUMEN
El estudio explora las diferencias, según género, en la valoración de los atributos del pisco, una bebida alcohólica tradicional de Chile. Mediante la técnica de Análisis Conjunto se analizaron las respuestas de 120 consumidores de la Región de Coquimbo (Chile), sobre cuatro atributos (sabor, tipo de fabricación, aroma y precio), revelando diferencias significativas en la apreciación de las características organolépticas y del precio. Se concluye que, independientemente del sexo, se prefiere un pisco de tipo artesanal de precio bajo, y que las diferencias se generan en el sabor y aroma, donde las mujeres prefieren piscos de sabor dulce y aroma a uva, mientras que los hombres; piscos de sabor fuerte y aroma a madera. Se espera que los hallazgos del estudio ayuden a los productores de pisco a focalizar sus estrategias de comercialización, así como a superar la poca información existente respecto de las preferencias del consumidor local de pisco.
\end{abstract}

Palabras clave: Preferencias del consumidor, bebida alcohólica, análisis conjunto, denominación de origen, Chile.

ABSTRACT

The study explores the gender differences in the evaluation of the attributes of pisco, a traditional alcoholic beverage from Chile. Through the Conjoint Analysis technique, the responses of 120 consumers in the Coquimbo Region (Chile) were analyzed, about four attributes (taste, type of manufacturing, aroma and price), revealing significant differences in the appreciation of the organoleptic characteristics and the price. It has concluded that, regardless of sex, a pisco of a low-price craft manufacturing type is preferred, and that the differences was generated in the flavor and aroma, where women prefer piscos with a sweet flavor and a grape aroma, while men prefer piscos with a strong flavor and wood aroma. We hoped that the findings of the study would help pisco producers to focus their marketing strategies, as well as to overcome the little information available regarding the preferences of the local pisco consumer.

Key words: Preferences of consumer, alcoholic beverage, conjoint analysis, designation of origin, Chile.

\section{Introducción}

En el periodo 2010-2016, según la Asociación Gremial de Fabricantes y Distribuidores de Licores y Bebidas Espirituosas de Chile (Aflechi), el consumo de destilados en Chile disminuyó en un $13 \%$, pasando de un volumen de ventas de 68,3 millones de litros, en el 2010 a 59,1 millones de litros durante el 2016.
Dicho contexto, no ha sido indiferente para el caso del pisco, cuyo consumo también se redujo cerca de un 4\% para el periodo, pasando de 2,03 a 1,9 litros per cápita (Paleo, 2017).

El pisco es un aguardiente de uva, elaborado en las regiones chilenas de Atacama y Coquimbo. Fue delimitado como Denominación de Origen (DO) en 1931 y representa de acuerdo a Lacoste et al. (2014)

\footnotetext{
${ }^{1}$ Departamento de Ciencias Económicas y Empresariales de la Universidad de La Serena, Amunátegui s/n, La Serena, Chile.

* Autor para correspondencia: saraya@userena.cl
}

Fecha de Recepción: 10 septiembre, 2018.

Fecha de Aceptación: 04 noviembre, 2018.

DOI: http://dx.doi.org/10.4067/S0718-34292018005002201. Publicado en línea: 11-marzo-2019. 
una de las experiencias más exitosas de DO del Cono Sur de América. La actual Ley de Alcoholes $\mathrm{N}^{\circ}$ 18455 de Chile deja claramente establecida que esta denominación queda reservada para el aguardiente producido y envasado para consumo en las Regiones de Atacama y Coquimbo, elaborado por destilación de vino genuino potable, proveniente de las variedades de vides que determine el reglamento, plantadas en dichas Regiones.

La baja paulatina del consumo de pisco en Chile, fruto del mayor protagonismo del vino y de la cerveza (Pradel, 2017), es particularmente preocupante para la Región de Coquimbo, pues es la principal zona pisquera del país al contar con cerca del $95 \%$ de la superficie de vides pisqueras (Buzzetti, 2017), en un sector productivo que se estima genera entre $30 \mathrm{mil}$ y 45 mil empleos (El Observatodo, 2016). Ante tal situación, nace la intención de estudiar las preferencias del consumidor de pisco para identificar cuáles son los atributos que influyen en su proceso de decisión de compra.

En Chile la evidencia empírica relacionada con la influencia de atributos diferenciadores en el comportamiento de compra y actitudes de los consumidores de pisco es escasa, no encontrándose estudios particulares que examinen las preferencias de los consumidores en base a modelos de valoración multiatributos o a tipologías específicas de segmentación del consumidor. Generalmente, las investigaciones se centran en el estudio del vino, donde es posible encontrar múltiples casos de estudio bajo diversos enfoques y metodologías de análisis (Baglyas y Sugatagi, 2011; Bernabéu et al., 2011; Cerda et al., 2010; De-Magistris et al., 2014; Farías y Fistrovich, 2016).

En este sentido, la presente investigación busca contribuir con el estudio de las preferencias de los consumidores por atributos diferenciadores para el pisco como una forma de proveer información de utilidad para las estrategias comerciales de los productores locales, enfocándose en explorar las diferencias de consumo entre hombres y mujeres. Énfasis que se justifica en la mayor participación de la mujer en el mercado laboral (INE, 2015) y su irrupción creciente en el consumo de bebidas alcohólicas (Garcés, 2015).

Específicamente, el estudio busca responder las siguientes preguntas: ¿Cuáles son los principales atributos buscados por los consumidores de pisco?, ¿Cuál es el peso relativo del precio en la decisión de compra?, ¿Existen diferencias significativas en la valoración de los atributos del pisco según género?
Dado lo anterior, los objetivos planteados son, primero, determinar cuáles son los atributos más relevantes para los consumidores de pisco de la región Coquimbo y, segundo, conocer si existen diferencias significativas según género.

El trabajo se estructura en cuatro acápites: i) antecedentes teóricos básicos sobre el estudio de las preferencias en destilados; ii) metodología utilizada; iii) resultados obtenidos; $y$ iv) conclusiones.

\section{Antecedentes teóricos}

Un factor clave de la demanda efectiva de un bien o servicio corresponde a la preferencia del consumidor, concepto que comprende al conjunto de elementos subjetivos del individuo que lo llevan a elegir determinados bienes y servicios que desea comprar (Reynolds, 2013). Precisamente, en el diseño de un modelo formal del comportamiento del consumidor el objetivo es caracterizar la elección de consumo donde sea indiscutible la preferencia por aquellos bienes que otorgan satisfacción o bienestar, considerando su restricción o límite presupuestario (Gil y Ríos, 2016). Así los productores pueden ofrecer bienes y servicios enfocados en satisfacer las necesidades expresadas en dichas preferencias. Adicionalmente, la posible creación de valor al incorporar ciertos atributos a los productos es una necesidad para las empresas que quieran mantenerse en mercados cada vez más competitivos y globalizados (Gil y Ibarra, 2014), donde la industria del pisco no es la excepción.

Desde el punto de vista económico, la teoría de la utilidad, representa una contribución fundamental para el estudio de las preferencias del consumidor. Esta concibe el concepto del valor como utilidad, es decir, el grado (medida) en que un satisfactor resuelve una necesidad.

Entre las numerosas técnicas existentes para la medición de la utilidad sobresalen las técnicas de las preferencias declaradas, un grupo de métodos que analiza la manera en que un individuo actuaría (selecciona o jerarquiza) frente a una serie de opciones hipotéticas que le son presentadas. Y es, precisamente, el Análisis Conjunto una de las técnicas de análisis multivariante más usado en el campo del estudio de las preferencias del consumidor. En ella el investigador representa productos o servicios a través de un conjunto de atributos y niveles con el objetivo de medir el nivel de utilidad que el usuario le asigna, considerando, por lo general, que la preferencia de una alternativa es una función aditiva de las utilidades 
parciales (part-worths) que la constituyen (Romero y Terán, 2011). Su aplicación en el mundo tiene una destacada trayectoria, encontrándose diversas investigaciones respecto a su evolución y uso en un país o región (Araya-Pizarro et al., 2018)

En el campo del estudio de las preferencias por atributos del pisco, las investigaciones son escasas y se centran, primordialmente, en examinar la situación del mercado e industria del pisco (Banfi, 2010; Higuchi et al., 2009); a estudios sobre su denominación de origen (Cofré et al., 2016; Lacoste et al., 2014); y a factores específicos que influyen en su intención de compra (Pipoli de Azambuja y García-Arrizabalaga, 2017).

Mientras que estudios concretos relativos al análisis de atributos por género se pueden encontrar en otras bebidas alcohólicas, principalmente vino. En ellos se destaca la importancia para las mujeres de atributos como: etiquetado del vino, región de procedencia, marca y medallas. Por ejemplo, Atkin, et al. (2007) aplicaron un cuestionario a 1374 consumidores de vino en Estados Unidos (497 hombres y 877 mujeres), encontrando que las etiquetas son significativamente más importantes para las mujeres y que si bien la región vitivinícola es muy relevante para ambos géneros, las mujeres se fijan más en las medallas y premios del producto. Forbes (2012), por su parte, encontró que el género influye significativamente en la utilización por parte del consumidor de vino de la aplicación de precio de descuentos y la referencia de la región de origen. Atkin y Sutanonpaiboon (2007) realizaron un estudio multinacional sobre las preferencias de vino según género, detectando que el patrón de preferencias de género de los consumidores de vino en esos países era bastante constante, lo que indicaba que el género era una base sobresaliente para segmentar el mercado del vino. Finalmente, Barber (2006), demostró que el diseño de las etiquetas y el sellado de las botellas de vino son trascendentes para los encuestados y que la autoconfianza es un factor importante para el género, donde las mujeres entre 31 y 40 años fueron las que mostraron mayor interés por comprar vino.

\section{Metodología}

Se realizó una encuesta personal a una muestra de 120 consumidores de pisco, cuyo número se obtuvo mediante la fórmula de muestreo probabilístico proporcional con afijación simple según la población de la Región de Coquimbo al Censo de 2002, 603 210 habitantes, considerando un $90 \%$ de confianza y un 7,51\% de error de estimación, con proporciones de p y q iguales a 0,5. Como instrumento de recolección de datos se aplicó un cuestionario con nueve preguntas bajo una escala Likert de 5 puntos (donde 1: total desacuerdo, 2: desacuerdo, 3: indiferente, 4: acuerdo y 5 : total acuerdo), sobre los factores que afectan el comportamiento de compra del pisco: el sabor, el precio, la marca, aroma, tipo de fabricación, calidad, experiencia previa, tiempo de añejado y presentación). Además se agregaron dos preguntas de caracterización de los encuestados: género y edad. También, la encuesta contó con un apartado para medir las preferencias del consumidor en base a 9 opciones del producto (hipotéticos) que el encuestado debió jerarquizar del más (1) al menos preferido (9). El instrumento se aplicó en supermercados de las ciudades de La Serena, Coquimbo y Ovalle, entre junio y julio de 2017, posterior a su validación mediante un pretest.

Para determinar la importancia de los atributos del pisco en la decisión de compra, según el sexo del encuestado, se utilizó análisis conjunto (AC) que corresponde a una técnica multivariante basada en la premisa de que los consumidores evalúan el valor de un producto/servicio (real o hipotético) combinando el valor individual que le reporta cada atributo. Dada la valoración que un conjunto de individuos hace de determinadas configuraciones alternativas de producto, se puede deducir la importancia de los atributos que configuran estas alternativas y de sus respectivos niveles (Hair et al.). Los atributos considerados para el estudio fueron cuatro: el sabor, el aroma, el tipo de fabricación y el precio. Los niveles definidos para el sabor fueron: fuerte, suave y dulce; en aroma se especificó: uva, pasas; en tipo de fabricación se planteó: artesanal e industrial; y en precio: USD \$ 10 (bajo), USD\$ 20 (medio) y USD\$ 30 (alto), correspondientes a precios de mercado del periodo de aplicación del instrumento.

En base a los atributos y niveles determinados se obtuvieron 9 combinaciones (tarjetas) identificadas alfabéticamente, desde la letra A hasta la letra I, tal como se muestra en la Tabla 1.

La función de preferencia correspondió al modelo aditivo, cuya representación conceptual fue la siguiente:

$$
U=U_{\text {sabor }_{i}}+U_{\text {fabricacion }_{j}}+U_{\text {aroma }_{k}}+U_{\text {precio }_{l}}+\text { constante }
$$


Por lo tanto, el modelo formulado para el análisis conjunto fue el siguiente:

$$
U_{t}=\alpha+\beta_{1} * x_{1 i}+\beta_{2} * x_{2 j}+\beta_{3} * x_{3 k}+\beta_{4} * x_{4 l}+e_{t}
$$

Tabla 1. Listado de tarjetas para la medición de las preferencias por el pisco

\begin{tabular}{ccccc}
\hline $\begin{array}{c}N^{\circ} \text { de } \\
\text { tarjeta }\end{array}$ & Sabor & Fabricación & Precio & Aroma \\
\hline A & Fuerte & Industrial & Bajo (USD\$ 10) & Uva \\
\hline B & Fuerte & Artesanal & Alto (USD\$ 30) & Pasas \\
\hline C & Suave & Artesanal & Bajo (USD\$ 10) & Pasas \\
\hline D & Suave & Artesanal & Medio (USD\$ 20) & Uva \\
\hline E & Suave & Industrial & Alto USD\$ 30) & Madera \\
\hline F & Dulce & Artesanal & Bajo (USD\$ 10) & Madera \\
\hline G & Dulce & Artesanal & Alto (USD\$ 30) & Uva \\
\hline H & Fuerte & Artesanal & Medio (USD\$ 20) & Madera \\
\hline I & Dulce & Industrial & Medio (USD\$ 20) & Pasas \\
\hline
\end{tabular}

Nota: Valores en dólares. Tipo de cambio de pesos chilenos por dólar americano observado es de 600 .

En la ecuación, $\mathrm{U}_{\mathrm{t}}$ representa el orden de preferencia establecido por el t-ésimo individuo encuestado, $\mathrm{x}_{1 \mathrm{i}}$ es la variable sabor $\left(x_{11}=\right.$ fuerte, $x_{12}=$ suave y $x_{13}=$ dulce), $\mathrm{x}_{2 \mathrm{j}}$ representa el tipo de fabricación ( $x_{21}=$ artesanal y $x_{22}=$ industrial), $\mathrm{x}_{3 \mathrm{k}}$ es el sabor $\left(x_{31}=\right.$ uva, $x_{32}=$ pasas y $x_{33}=$ madera) y $\mathrm{x}_{41} \mathrm{el}$ precio $\left(x_{41}=\mathrm{USD} \$ 10, x_{42}=\mathrm{USD} \$ 20\right.$ y $\left.x_{43}=\mathrm{USD} \$ 30\right)$, $\alpha$ es la constante de la regresión; $\beta_{1}, \beta_{2}, \beta_{3}$ y $\beta_{4}$ son los valores de utilidad parciales asociados a los niveles de cada atributo, y $\varepsilon_{\mathrm{t}}$ es el error de estimación.

Para la recogida de datos se usó el procedimiento de perfil total, para lo cual se elaboraron las nueve tarjetas con su respectiva especificación de atributos y niveles. Para el atributo precio se estableció una relación lineal inversa, debido a que generalmente a menor precio la utilidad o preferencia es mayor. $\mathrm{El}$ resto de los atributos fueron considerados como variables discretas. Para determinar la bondad de ajuste del modelo conjunto se usó el coeficiente de correlación de Pearson y se utilizó la Tau-B de Kendall para establecer si la ordenación estimada de los estímulos según la función propuesta corresponde con la ordenación real del encuestado.

Los resultados de las preguntas sobre los factores que afectan el comportamiento de compra del consumidor de pisco según sexo fueron analizados mediante Tablas de Contingencia, ANOVA y el estadístico Chi Cuadrado. La fiabilidad de las ddsmisdió a través del cómputo del coeficiente Alfa de Cronbach, obteniendo un resultado aceptable $(0,737)$.
Todos los cálculos efectuados, tanto para el análisis de las preferencias (Análisis Conjunto) como para el examen de las tablas de contingencia y de frecuencias, fueron conseguidos mediante el software PASW Statistics 18.0 para Windows.

\section{Resultados}

Este apartado se divide en cuatro secciones: i) factores que influyen en la decisión de compra según género, ii) preferencias de las mujeres, iii) preferencias de los hombres, y iv) contraste de las preferencias por sexo.

\section{Factores que influyen en la decisión de compra según género:}

La muestra de encuestados estuvo conformada por 60 mujeres y 60 hombres, cuya distribución etaria, que se presenta en la Tabla 2, mostró que la mayoría de los consumidores, tienen entre 20 y 40 años.

Tabla 2. Frecuencias absolutas y relativas por rango etario y sexo

\begin{tabular}{ccccccc}
\hline \multirow{2}{*}{ Rango de edad } & \multicolumn{2}{c}{ Global } & \multicolumn{2}{c}{ Mujeres } & \multicolumn{2}{c}{ Hombres } \\
\cline { 2 - 7 } & $\mathrm{N}$ & $\%$ & $\mathrm{~N}$ & $\%$ & $\mathrm{~N}$ & $\%$ \\
\hline Menos de 20 años & 27 & $23 \%$ & 12 & $20 \%$ & 15 & $25 \%$ \\
\hline 20 y 40 años & 88 & $73 \%$ & 44 & $73 \%$ & 44 & $73 \%$ \\
\hline Más de 40 años & 5 & $4 \%$ & 4 & $7 \%$ & 1 & $2 \%$ \\
\hline
\end{tabular}

La Tabla 3 muestra el análisis de los resultados (media \pm desviación estándar) vinculados a los factores influyentes en la decisión de compra, lo que reveló que:

- En el caso de las mujeres, compran pisco en base a experiencias anteriores $(4,23 \pm 0,67)$, donde el sabor $(4,05 \pm 1,00)$ y el conocimiento de la marca $(4,05 \pm 1,03)$ son fundamentales para su elección. A su vez, consideran que el pisco artesanal tiene una calidad superior a los demás piscos del mercado $(3,80 \pm 0,95)$ y que la presentación del producto influye en la decisión de compra $(3,75 \pm 1,00)$.

- En el caso de los hombres, compran pisco en base a experiencias pasadas $(4,07 \pm 1,04)$ y relacionan positivamente el precio del pisco con 
su calidad $(3,80 \pm 1,05)$. Otorgan relevancia a la presentación del producto $(3,78 \pm 1,03)$ y admiten que el conocimiento de la marca es un factor influyente en su decisión de compra $(3,78 \pm 1,06)$.

El contraste de los factores comportamentales según sexo, evidenció diferencias significativas $(p<0,05)$ en las características organolépticas del pisco: sabor $\left(\chi^{2}=12,85 ; p=0,01\right)$ y aroma $\left(\chi^{2}=10,05\right.$; $p=0,04)$. Además, las pruebas de diferencias de medias confirmaron (95\% de confianza) que las mujeres, tanto en el sabor $(\mathrm{F}=6,81 ; p=0,010)$ como en el aroma $(\mathrm{F}=3,99 ; p=0,048)$, otorgan una valoración mayor que los hombres. La Figura 1, muestra las brechas detalladas anteriormente.

Tabla 3. Promedios y desviación estándar agregado y según género

\begin{tabular}{lcccc}
\hline \multirow{2}{*}{ Pregunta } & \multicolumn{2}{c}{ Media } & \multicolumn{2}{c}{ Desviación estándar } \\
\cline { 2 - 6 } & Mujer & Hombre & Mujer & Hombre \\
\hline El sabor del pisco es predominante a la hora de elegir. & 4,05 & 3,52 & 0,99 & 1,23 \\
\hline $\begin{array}{l}\text { La opinión del entorno es importante a la hora de decidir } \\
\text { qué pisco comprar. }\end{array}$ & 3,42 & 3,50 & 1,21 & 1,16 \\
\hline Mientras más elevado es el precio, más alta es la calidad. & 3,60 & 3,80 & 1,12 & 1,05 \\
\hline El conocimiento de la marca influye en la decisión compra. & 4,05 & 3,77 & 1,03 & 1,06 \\
\hline El aroma determina si volver a comprar o no un pisco. & 3,60 & 3,20 & 1,01 & 1,18 \\
\hline Compra pisco en base a experiencias anteriores. & 4,23 & 4,07 & 0,67 & 1,04 \\
\hline $\begin{array}{l}\text { El pisco artesanal tiene una calidad superior a la de los } \\
\text { demás piscos del mercado. }\end{array}$ & 3,80 & 3,55 & 0,95 & 0,91 \\
\hline El tiempo de añejado del pisco es importante para su elección. & 3,62 & 3,55 & 1,12 & 1,16 \\
\hline La presentación del pisco importante a la hora de elegir. & 3,75 & 3,77 & 1,00 & 1,03 \\
\hline
\end{tabular}

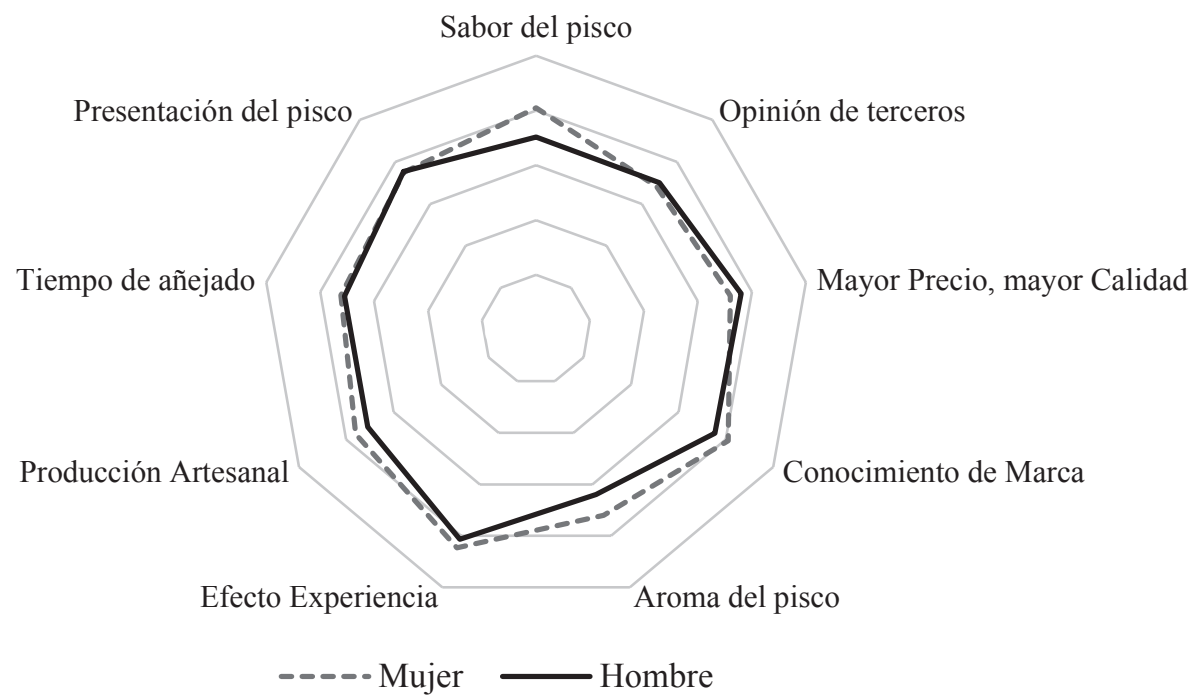

Figura 1. Factores que influyen en la decisión de compra según género 


\section{Preferencias de las mujeres:}

El reporte de la estimación del modelo de Análisis Conjunto, para el caso de las mujeres, se resume en la Tabla 4 donde se observa la importancia relativa (\%) que conceden a los distintos atributos y las utilidades parciales de sus correspondientes niveles.

Tabla 4. Resultados agregados de la importancia relativa (\%) de los atributos y las utilidades parciales para las mujeres

\begin{tabular}{|c|c|c|c|}
\hline Atributos & $\begin{array}{l}\text { Importancia } \\
\text { relativa (\%) }\end{array}$ & Niveles & $\begin{array}{l}\text { Utilidad Parcial } \\
\text { (Part-worths) }\end{array}$ \\
\hline \multirow{3}{*}{ Sabor } & \multirow{3}{*}{31,32} & Fuerte & $-0,672$ \\
\hline & & Suave & 0,272 \\
\hline & & Dulce & 0,400 \\
\hline \multirow{2}{*}{ Fabricación } & \multirow{2}{*}{15,58} & Artesanal & 0,313 \\
\hline & & Industrial & $-0,313$ \\
\hline \multirow{3}{*}{ Aroma } & \multirow{3}{*}{27,17} & Uva & 0,122 \\
\hline & & Pasas & $-0,117$ \\
\hline & & Madera & $-0,006$ \\
\hline \multirow{3}{*}{ Precio } & \multirow{3}{*}{25,93} & Bajo (USD\$ 10) & $-0,400$ \\
\hline & & Medio (USD\$20) & $-0,800$ \\
\hline & & Alto (USD\$ 30) & $-1,200$ \\
\hline Constante: & 5.696 & $\begin{array}{l}\text { R de Pearson: } \\
0,945\end{array}$ & $\begin{array}{r}\text { Tau-B de Kendall: } \\
0,833\end{array}$ \\
\hline
\end{tabular}

*** Nivel de significación $(\mathrm{p} \leq 0,001)$

A partir de los resultados obtenidos sobre la importancia relativa de los atributos, se puede afirmar que el sabor es el atributo más relevante en la formación de preferencias de los consumidores de pisco, con una importancia relativa del $31,32 \%$, seguido por el atributo aroma (27,17\%); en tercer lugar, el precio, con una importancia de $25,93 \%$, y por último el atributo tipo de fabricación con una valoración de 15,58\%.

Las medidas de bondad de ajuste del modelo ( $\mathrm{R}$ de Pearson y Tau-B de Kendall) mostraron un ajuste alto con coeficientes muy significativos $(\mathrm{p} \leq 0,001)$.

La función de utilidad agregada para el conjunto de mujeres, tuvo por tanto la siguiente forma:

$\mathrm{U}=[-0,672$ Fuerte $+0,272$ Suave $+0,400$ Dulce $]+$ [0,313 Artesanal $-0,313$ Industrial $]+[0,122$ Uva $0,117$ Pasas $-0,006$ Madera $]+[-0,400($ US\$ 10) $0,800(\mathrm{USD} \$ 20)-1.200(\mathrm{USD} \$ 30)]+5.696$

Dentro del "Sabor", el nivel Dulce constituyó el factor más relevante en la formación de las preferencias, siendo el valor de su utilidad media relativa positivo de 0,4 , superior al sabor suave $(0,272)$ y fuerte $(-0,672)$, así, cuando el pisco tiene sabor dulce mayor es su nivel de apreciación y utilidad por parte de las mujeres encuestadas.

En relación al atributo "Tipo de fabricación”, el pisco elaborado de modo artesanal es más valorado que el de fabricación industrial, siendo su utilidad parcial positiva $(0,313)$. Por tanto, cuanto menos industrializado es el proceso de elaboración del pisco, y más aún si está fabricado utilizando técnicas artesanales, mayor es la utilidad y satisfacción por parte de los consumidores.

Respecto al atributo "Aroma", el nivel uva, es el más determinante en la función de utilidad derivada de la preferencia del pisco, siendo positiva su utilidad parcial $(0,122)$ y superior a las utilidades del aroma a madera $(-0,006)$ o a pasas $(-0,117)$.

Por último, en cuanto al atributo "Precio", sus utilidades parciales tienen el signo esperado según la teoría económica, demostrando que la utilidad marginal de una alternativa disminuye cuando su precio es mayor.

De los resultados anteriores se puede afirmar que el "pisco más preferido" por las entrevistadas, según los atributos y niveles estudiados, sería el de sabor dulce, de tipo artesanal, aroma a uvas y de bajo precio. Mientras que el "pisco menos preferido" es el de sabor fuerte, de tipo industrial, aroma a pasas y de alto precio.

\section{Preferencias de los hombres:}

Los resultados de la estimación del modelo de Análisis Conjunto, para el caso de los hombres, se reflejan en la Tabla 5. En ella se aprecia que el sabor es el atributo más relevante en la formación de preferencias de los consumidores de pisco $(29,95 \%)$, seguido por el atributo precio $(29,76 \%)$; en tercer lugar, el aroma $(24,3 \%)$, y finalmente el atributo tipo de fabricación $(15,98 \%)$.

Las medidas de bondad de ajuste del modelo mostraron un ajuste aceptable, con coeficientes muy significativos $(\mathrm{p} \leq 0,001)$.

El modelo estimado de utilidad agregada para los hombres, fue el siguiente:

$\mathrm{U}=[0,239$ Fuerte $-0,178$ Suave $-0,061$ Dulce $]+$ [0,054 Artesanal -0,054 Industrial $]+[0,094$ Uva $0,194$ Pasas + 0,100 Madera $]+[-0,219($ US\$ 10) 0,439 (USD\$ 20) $-0,658$ (USD\$ 30)] + 5.421

Dentro del "Sabor", el nivel Fuerte fue el factor preferido, siendo el valor de su utilidad media relativa 
Tabla 5. Resultados agregados de la importancia relativa (\%) de los atributos y las utilidades parciales para los hombres

\begin{tabular}{|c|c|c|c|}
\hline Atributos & $\begin{array}{l}\text { Importancia } \\
\text { relativa }(\%)\end{array}$ & Niveles & $\begin{array}{l}\text { Utilidad Parcial } \\
\text { (Part-worths) }\end{array}$ \\
\hline \multirow{3}{*}{ Sabor } & \multirow{3}{*}{29,95} & Fuerte & 0,239 \\
\hline & & Suave & $-0,178$ \\
\hline & & Dulce & $-0,061$ \\
\hline \multirow{2}{*}{ Fabricación } & \multirow{2}{*}{15,98} & Artesanal & 0,054 \\
\hline & & Industrial & $-0,054$ \\
\hline \multirow{3}{*}{ Aroma } & \multirow{3}{*}{24,30} & Uva & 0,094 \\
\hline & & Pasas & $-0,194$ \\
\hline & & Madera & 0,100 \\
\hline \multirow{3}{*}{ Precio } & \multirow{3}{*}{29,76} & Bajo (USD\$ 10) & $-0,219$ \\
\hline & & Medio (USD\$ 20) & $-0,439$ \\
\hline & & Alto (USD\$ 30) & $-0,658$ \\
\hline Constante: & 5.421 & $\begin{array}{l}\text { R de Pearson: } \\
0,734\end{array}$ & $\begin{array}{l}\text { Tau-B de } \\
\text { Kendall: } 0,556\end{array}$ \\
\hline
\end{tabular}

*** Nivel de significación $(p \leq 0,001)$

positivo de 0,239 , superior al sabor suave $(-0,178)$ y dulce $(-0,061)$. Ello refleja que, para los varones, el sabor suave es el nivel menos importante.

En cuanto al atributo "Tipo de fabricación", el pisco elaborado de modo artesanal es más valorado que el de fabricación industrial, siendo su utilidad parcial positiva $(0,054)$.

Respecto al atributo "Aroma”, el nivel Madera, es el más influyente en la función de utilidad derivada de la preferencia del pisco, siendo positiva su utilidad parcial $(0,100)$ y superior a las utilidades del aroma a uva $(0,094)$ o a pasas $(-0,194)$.

Finalmente, en relación al atributo "Precio", sus utilidades parciales registraron el signo esperado, confirmando que la utilidad disminuye ante precios más altos.

De los resultados anteriores se puede afirmar que el "pisco más preferido" por los hombres, según los atributos y niveles estudiados, sería el de sabor fuerte, de tipo artesanal, aroma a madera y de bajo precio. Mientras que el "pisco menos preferido" es el de sabor suave, de tipo industrial, aroma a pasas y de alto precio.

\section{Contraste de las preferencias por sexo:}

El estudio comparativo de los atributos del pisco, mostró coincidencias en la jerarquía del atributo más y menos preferido para las mujeres y los hombres: el "sabor" (mujeres: 31,32\% y hombres: 29,95\%) y el "tipo de fabricación" (mujeres: 15,58\% y hombres: 15,98\%), respectivamente. Sin embargo, se observó que las mujeres aprecian más que los hombres, las características de aroma (mujeres: $27,17 \%$ vs hombres: $24,30 \%$ ), mientras que los hombres, por su parte, dan mayor importancia al atributo precio que las mujeres (hombres: $29,76 \%$ vs mujeres: $25,93 \%$ ). Véase la Figura 2.

La Figura 3, contiene el análisis comparativo para cada uno de los niveles por atributos del pisco, el cual revela que tanto hombres como mujeres prefieren un pisco de "fabricación artesanal" (hombres: 0,05 y mujeres: 0,31 ) y de "precio bajo" (hombres: $-0,22$ y mujeres: -0,40). En cuanto a las diferencias, se observa que los hombres dan una mayor utilidad a un "sabor fuerte" $(0,24)$ y las mujeres a un "sabor dulce" $(0,40)$. A su vez, las mujeres otorgan una utilidad mayor a un

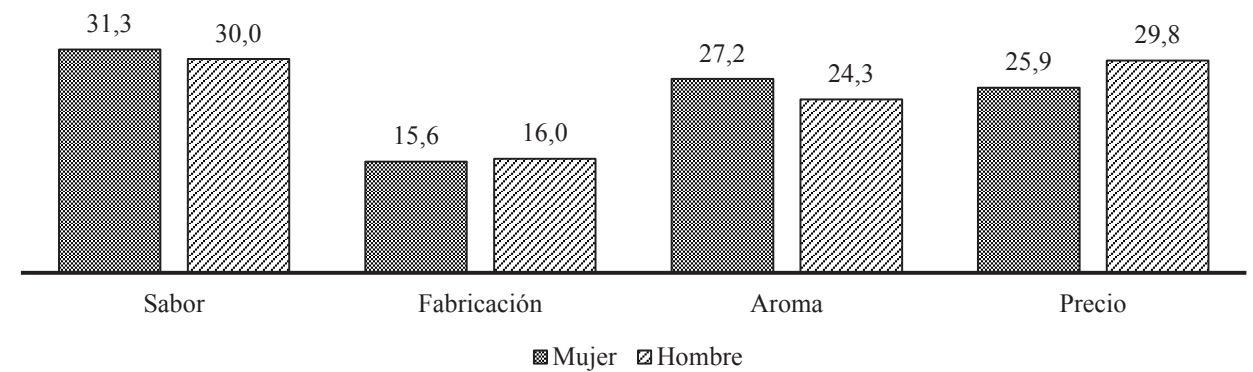

Figura 2. Contraste de la valoración de atributos del pisco según género 


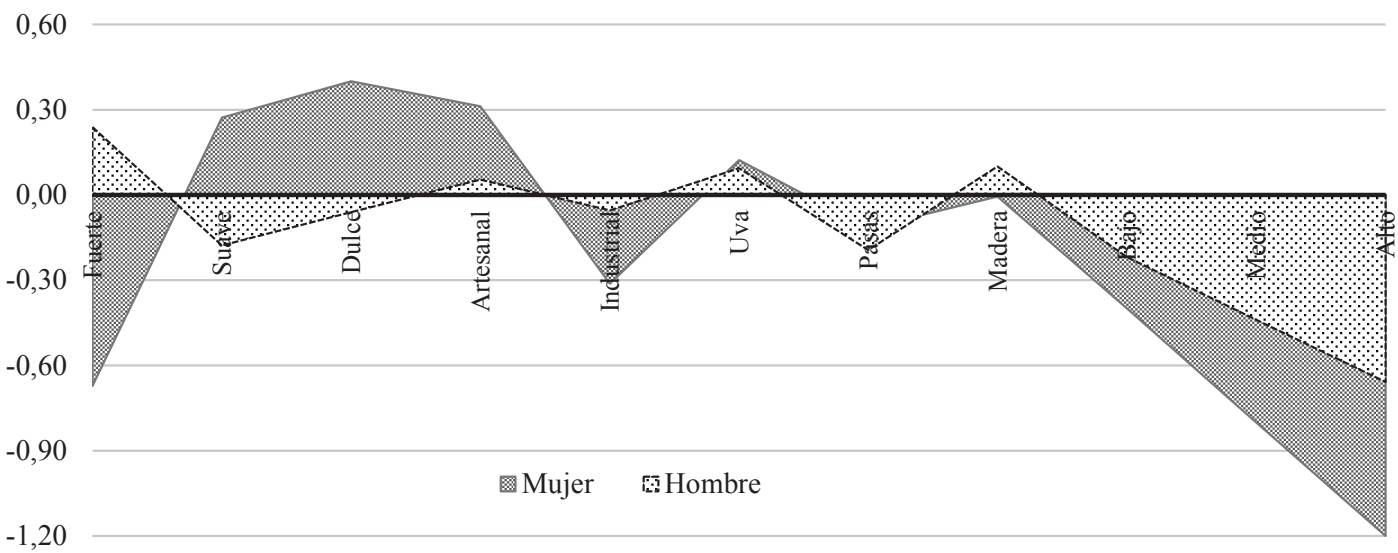

Figura 3. Contraste de niveles de utilidad del pisco según género

pisco con "aroma a uva" $(0,12)$, y los hombres a un pisco con "aroma a madera" $(0,10)$.

\section{Conclusiones}

La presente investigación, planteó como propósito explorar las diferencias de género en la valoración de los atributos del pisco, una bebida alcohólica tradicional de Chile. Mediante la aplicación de la metodología del análisis conjunto (Conjoint Analysis), a una muestra de 120 bebedores de pisco de la Región de Coquimbo, se pudo concluir que existen diferencias significativas en la valoración de las características organolépticas (sabor y aroma), que son más importantes para las mujeres, y el precio, que es más relevante para los hombres.

Se determinó, además que, independientemente del sexo, se prefiere un pisco de tipo artesanal de precio bajo, y que las diferencias se generan en los niveles de los atributos: sabor y aroma, donde las mujeres prefieren piscos de sabor dulce y aroma a uva, mientras que los hombres; piscos de sabor fuerte y aroma a madera.

El análisis conjunto demostró que las diferencias en sabor y aroma, abren oportunidades para los productores locales innoven en el producto, desarrollando alternativas de pisco artesanal que para el caso de los hombres se concentren en generar destilados con alto grado alcohólico y de sabor fuerte. Y, para el caso de las mujeres, elaborar alternativas con mayor concentración de azúcar.

Cabe destacar, que el presente estudio, consistió en una primera aproximación sobre las preferencias del consumo de pisco bajo una perspectiva de género, por lo que empleó un tamaño de muestra limitado a niveles de confiabilidad y error aceptables. Se sugiere corroborar los resultados, utilizando una muestra de mayor tamaño, que considere extender el alcance dentro de la Región. Esto con el fin de obtener una idea complementaria y más acabada sobre la estructura de preferencias de los consumidores regionales de pisco. En el mismo escenario, se torna conveniente analizar las preferencias de los consumidores del país, a fin de validar y comparar los resultados obtenidos.

Por último, como dirección para futuras investigaciones, se plantea estimar la disposición a pagar, de mujeres y hombres, por un pisco artesanal, así como aplicar la técnica de análisis conjunto a temáticas relacionadas como, por ejemplo, el examen de las preferencias por otras bebidas alcohólicas, que han crecido fuertemente en el mercado local, como el ron o el whisky (Pérez-Cueto, 2017).

\section{Literatura Citada}

Araya-Pizarro, S.; Varas-Madrid, C.; Rojas-Escobar, L.

2018. Atributos Preferidos por los Estudiantes de Ingeniería Comercial en la Enseñanza de la Asignatura Administración de Empresas. Formación universitaria, 11 (1): 77-86.
Atkin, T.; Nowak, L.; García, R.

2007. Women wine consumers: Information search and retailing implications. International Journal of Wine Business Research, 19 (4): 327-339. 
Atkin, T.; Sutanonpaiboon, J.

2007. A Multinational Study of Gender Wine Preferences. A multinational study of gender wine preferences. In: Proceedings of the International DSI / Asia and Pacific DSI. 19p.

Baglyas, F.; Sugatagi G.

2011: Multivariate analysis in a wine market research in Hungary. Annals of Faculty Engineering Huneodara International Journal of Engineering, IX. Extra Fascicule: 235-238.

Barber, N.; Almanza, B.; Donovan, J.

2006. Motivational factors of gender, income and age on selecting a bottle of wine. International Journal of Wine Marketing, 18 (3): 218-232.

Buzzetti, C.

2017. Evolución de la producción y mercado del Pisco: noviembre de 2017. Odepa. Santiago, Chile. 13p.

Cerda, A.; Torres, M.; García, L.

2010. Preferencias y disposición a pagar por vinos orgánicos de parte de los consumidores de la región del Maule. Revista Panorama Socioeconómico, 28 (40): 60-71.

Cofre, C.; Núñez, E.; Mujica, F.; Lacoste, P.

2016. La guerra del pisco a través del estudio de los marbetes. Idesia (Arica), 34 (2): 25-34.

De-Magistris, M.; Groot, T.; Gracia A.; Albisu, L.

2014. Consumer preferences for wine in Spain: best-worst scaling methodology. Spanish Journal of Agricultural Research, 12 (3): 529-541.

El Observatodo.

2016. Regiones de Coquimbo y Atacama unidos en fortalecer proyección y producción de pisco. El Observatodo (región de Coquimbo). 13 de mayo de 2016. Disponible: http://www.elobservatodo.cl/noticia/politica/ regiones-de-coquimbo-y-atacama-unidos-en-fortalecerproyeccion-y-produccion-de-pisc. Consultado:03/dic/2017

Farías, P.; Fistrovic, B.

2016. Las preferencias del consumidor usando el método de máximas diferencias. Revista de Administração de Empresas, 56 (2): 138-151.

Forbes, S.

2012. The influence of gender on wine purchasing and consumption: An exploratory study across four nations, International Journal of Wine Business Research, 24 (2): 146-159.

Garcés, B.

2015. Consumo de alcohol en Chile crece $20 \%$ en dos años. Universidad San Sebastián (Santiago, Chile). 13 de julio de 2015. Disponible: http://www.uss.cl/blog/consumode-alcohol-en-chile-crece-20-en-dos-anos/.Consultado:03/ dic/2017.
Gil, I.; Ibarra, S.

2014. Incidencia del liderazgo en los factores críticos del éxito como estrategia competitiva empresarial. Dimensión Empresarial, 12 (2): 117-126.

Gil, E.O.; Ríos, H.F.

2016. Hábitos y preferencias de consumo. Consumo en estudiantes universitarios. Dimensión Empresarial, 14 (2): 55-72.

Hair, J.; Anderson, R; Tatham, R.; Black, W. 1999. Análisis Multivariante. Madrid: Prentice Hall.

Higuchi, A.; Yutaka, T.; Fukuda, S.

2009. An Analysis of the Current Situation of the Small Peruvian Pisco Producers in the Cañete Valley: A Case of Study among Associated Farmers vs Non-associated Ones. J. Fac. Agr., Kyushu Univ., 54 (2): 535-540.

Instituto Nacional de Estadísticas.

2015. Mujeres en Chile y mercado del trabajo. INE. Santiago, Chile. 136p.

Lacoste, P.; Briones, F.; Jiménez, D.; Rendón, B.

2014. La Denominación de Origen Pisco en Chile: algunos problemas nacionales e internacionales. Idesia (Arica), 32 (2): 47-56.

Paleo, D.

2017. Ventas de pisco registran alza en el último año, pero consumo ha disminuido 4\% en seis años. Diario el Día (La Serena, Chile). 23 de febrero 2017. Disponible en: http://www. diarioeldia.cl/economia/pisco/ventas-pisco-registran-alzaen-ultimo-ano-pero-consumo-ha-disminuido-4-en-seis-anos. Consultado:15/dic/2017

Pérez-Cueto, C.

2017. Consumo de whisky en Chile desplaza al ron. Diario La Tercera (Santiago, Chile). 12 de diciembre 2017. Disponible en: http://www2.latercera.com/noticia/consumo-whiskychile-desplaza-al-ron/.Consultado: 05/mar/2018.

Pipoli de Azambuja, G.; García-Arrizabalaga, I.

2017. Análisis de las variables que se asocian con la intención de compra del pisco del Perú. Multidisciplinary Business Review, 10 (1): 54-61.

Pradel, D.

2017. Consumo de destilados cae $13 \%$ en seis años: Pisco y ron son los productos más afectados. Diario El Mercurio (Santiago, Chile). 22 de febrero de 2017. Disponible en: http:// www.emol.com/noticias/Economia/2017/02/22/846135/ Consumo-de-destilados-cae-13-en-seis-anos-Pisco-y-ronson-los-productos-mas-afectados.html. Consultado:03/ dic/2017.

Reynolds, L.

2013. Percepciones y preferencias del consumidor de palmito fresco. Caso: unión de asociaciones de productores de plantines y palmito. Revista Perspectivas, (32), 57-100. 
